\title{
Studies on Chromogranin Levels in Human Blood
}

\author{
Ritsuji YAMADA, Jin SATO AND EIzo KOMIYA \\ Department of Clinical Pathology, Dokkyo University School \\ of Medicine, Mibu, Shimotsuga, Tochigi 321-02, Japan
}

\begin{abstract}
Synopsis
Considering that the determination of chromogranin levels in the blood for the diagnosis of pheochromocytoma will provide clinically useful information, an attempt was made to establish the serological assay system for blood chromogranin, and also chromogranin levels in the blood of normal subjects, patients with essential hypertension and a patient with pheochromocytoma were studied.

Chromogranin was isolated and purified from the granular internal fluid of the catecholamine storing vesicles of the pig adrenal glands. The antibodies against chromogranin and the chromogranin-sensitized sheep red blood cells were prepared; and chromogranin levels in the blood were determined by the passive hemagglutination inhibition reaction (PHI), using the microtiter system.

As it was observed in the determination of chromogranin levels in the blood by PHI that globulin fractions gave rise to nonspecific agglutination by this reaction system, the serum samples were pretreated by the salting-out method with ammonium sulfate into the albumin fraction, on which PHI was performed.

Chromogranin levels in the blood of 41 normal subjects and 12 patients with essential hypertension in terms of inhibition values of PHI were distributed chiefly in the range from $1: 20$ to $1: 80$. The level in the blood of one patient with pheochromocytoma was $1: 640$, whose titer was obviously higher than the titers in the blood of normal subjects and patients with essential hypertension. These evidences suggest that the determination of chromogranin levels in the blood will provide useful information for the diagnosis of pheochromocytoma.
\end{abstract}

The release of catecholamines from the adrenal medulla results from exocytosis of the catecholamine storing vesicles on stimulation of medulla cells (Robertis and Ferreira, 1957; Helle and Serck-Hanssen, 1975). It is also accepted that ATP and chromogranin contained in the vesicles are simultaneously released (Douglas et al., 1965; Banks, 1966). What are named by Blaschko et al. (1967) "chromogranins" are a fraction containing a variety of substances, which certainly contain dopamine- $\beta$-hydroxylase $(\mathrm{DBH})$. As the fraction was more

Received July 19, 1978. and more purified thereafter, chromogranin A, chromogranin S, and chromogranin VI-I were regarded as identical with each other in view of their molecular weights and amino acid composition, and were called "chromogranin" (Helle, 1970). This is a purified protein specifically affinitive for catecholamines (Smith and Kirschner, 1967).

Today, attempts are made by many investigators in the determination of enzymatic activity of DBH for the diagnosis of pheochromocytoma, besides the determination of catecholamines in blood and urine (Joh et al., 1974). However, investigators vary in evaluation because this enzyme 
occurs only in small amounts in chromogranin (Geffen et al., 1969; Smith and Kirschner, 1967), and also because there are many problems yet to be solved in the determination of its enzymatic activity (Yamada and Sato, 1977). On the other hand, the detection of sensitivity for it does not vary owing to various factors on the subject side but remains constant (Yamada et al., 1977), because chromogranin is released in far larger amounts as a result of exocytosis than $\mathrm{DBH}$, and also because it is not detected and isolated as a biological activity, as an enzyme, but done as a protein itself.

Paying attention to chromogranin which is thus simultaneously released with catecholamines and DBH, we made the study described hereunder. The serological assay system for chromogranin described in a previous paper (Yamada et al., 1978) was further examined, and modified so as to be capable of determining chromogranin in blood. Furthermore, the present paper describes the determination of chromogranin in the blood of normal subjects, patients with essential hypertension and a patient with pheochromocytoma, and also contains the discussion of its clinical significance.

\section{Materials and Methods}

Chromogranin, anti-chromogranin antiserum and chromogranin-sensitized sheep red blood cells were prepared by the methods described in a previous paper (Yamada et al, 1978). The medulla was collected from the fresh pig adrenal glands, and ground in $0.3 \mathrm{M}$ sucrose solution. It was then centrifugally separated by the continuous sucrose density gradient method, to collect the catecholamine storing vesicles. The vesicle membranes were broken, and the internal fluid was purified by DEAE cellulose column chromatography into chromogranin. The antibodies against chromogranin were produced by giving injection to the rabbit foot pads with chromogranin together with Freund's complete adjuvant. The chromograninsensitized sheep red blood cells were produced by first allowing tannic acid to act on the formalinized sheep red blood cells and then allowing chromogranin to bind to the cells.
Passive hemagglutination reaction and its inhibition reaction (PHI) were allowed to take place by the use of a microtiter system with a $1 \%$ mixture of normal rabbit serum with phosphate buffer saline solution (PBS), pH 6.9 as the reaction solution diluent.

The human serum for the determination of chromogranin was pretreated as described below prior to PHI. To $0.2 \mathrm{ml}$ of the serum was added $0.2 \mathrm{~m} l$ of saturated solution of ammonium sulfate to remove the precipitating globulin fraction. To the solution of ammonium sulfate to collect the albumin fraction, which was dialyzed against $0.14 \mathrm{M}$ saline to remove ammonium sulfate. A sufficient amount of PBS was then added to make $1 \mathrm{ml}$, and chromogranin was determined.

For the determination of catecholamines in urine, epinephrine and norepinephrine were determined on the 24-hrs urine sample acidified with hydrochloric: acid by THI method (Yamada, 1975).

\section{Results}

PHI was performed directly on the serum samples in order to determine chromogranin levels in blood, but no inhibition reaction took place at all. PHI was then made on the chromogranin-containing serum, but the serum proved unreactive. Therefore, PHI was performed on a mixture of PBS and the same amount of chromogranin as had been added to the serum, and a definite inhibition reaction took place. These findings suggest the occurence in the serum of a certain substance interfering with the PHI system. In which fraction this interfering substance occurs was studied by the salting-out method using ammonium sulfate, and it was found to occur in the globulin fraction. At the same time it was found that chromogranin occurred in the albumin fraction. For this reason, it was decided to pretreat the serum samples for the determination of chromogranin in blood into the albumin fraction by the salting-out method as described in the Methods prior to PHI.

In Fig. 1 are shown the results of determination by PHI of chromogranin in the blood of 41 normal subjects, 12 patients 
with essential hypertension and one patient with pheochromocytoma. Chromogranin levels in the blood of the normal subjects and the patients with essential hypertension occurred in the range from $1: 5$ to $1: 160$, but chiefly within the range from $1: 20$ to $1: 80$. However, the level

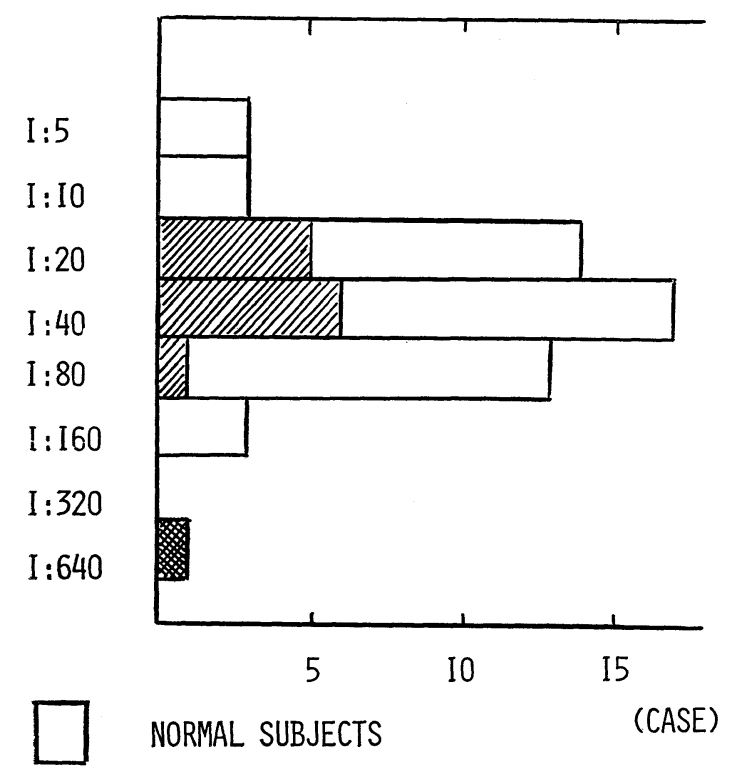

PATIENTS WITH ESSENTIAL HYPERTENSION

\section{PATIENT WITH PHEOCHROMOCYTOMA}

Fig. 1. Chromogranin levels in the blood of 41 normal subjects, 12 patients with essential hypertension and one patient with pheochromocytoma. Chromogranin levels were determined by Inhibition titer of passive hemagglutination reaction using anti-chromogranin and chromogranin-sensitized red cell. in the patient with pheochromocytoma was $1: 640$, which was evidently high, compared with the levels in the normal subjects (mean $1: 51,1$ SD range $1: 11-1: 91)$ and the patients with essential hypertension (mean $1: 35,1$ SD range $1: 18-1: 52$ ).

Table 1 shows alterations with the passage of time in catecholamines in the urine and chromogranin level in the blood of the patient with pheochromocytoma. As is clear from the urinary outputs of catecholamines, the release of catecholamines. in this patient was not so large for pheochromocytoma, but the finding that the chromogranin level in the blood of this patient at a certain stage, $26 \mathrm{July,}$ was as high as $1: 640$ calls for attention.

\section{Discussion}

No attempts have been made for the determination of chromogranin in the human serum to date. We have been studying serological quantitative analysis of chromogranin (Yamada and Sato, 1977; Yamada et al., 1977; Yamada et al., 1978). The antiserum used in the present study gives rise to a precipitin reaction with the chromogranin in the pig adrenal medulla and also exhibits a precipitin activity with the chromogranin in the human adrenal medulla (Yamada and Sato, 1977). We have already demonstrated the semiquantitative determination by PHI of the chromogranin released from pig adrenal medulla and the chromogranin eluted from the re-

Table 1. Catecholamines concentration in $24 \mathrm{hrs}$ urine and chromogranin level in the blood of the patient with pheochromocytoma.

\begin{tabular}{cccc}
\hline \multirow{2}{*}{ Date } & \multicolumn{2}{c}{$\begin{array}{c}\text { catecholamines concentration } \\
\text { in 24 hrs urine }(\mu \mathrm{g} / \text { day) }\end{array}$} & $\begin{array}{c}\text { chromogranin level in blood } \\
\text { (PHI titer) }\end{array}$ \\
\cline { 2 - 4 } 22, Jun & 39.5 & norepinephrine & $1: 40$ \\
22, July & 18.5 & 5.3 & $1: 40$ \\
23, July & 19.6 & 13.9 & $1: 640$ \\
26, July & 16.2 & 14.6 & 19.2 \\
\hline
\end{tabular}


sected tissue extract of pheochromocytoma (Yamada et al., 1978).

When PHI was performed directly on the serum samples in order to determine chromogranin levels in the blood by the PHI system, no inhibition reaction took place in spite of the fact that chromogranin was definitely contained in the serum. This phenomenon had been brought forth by a certain substance capable of nonspecifically agglutinating the sensitized sheep red blood cells, and it was found by the salting-out method that the substance occurred in the globulin fraction. As it was also revealed that chromogranin, when fractionated by the salting-out method, occurred in the albumin fraction, we successfully removed the substance interfering with the PHI system by means of fractionating the serum samples only into the albumin fraction by the salting-out method using ammonium sulfate.

When chromogranin levels in the blood of the normal subjects and patients with essential hypertension were studied by the modification of PHI, including such a pretreatment, it was found that chromogranin levels in the subjects and patients were, as shown in Fig. 1, chiefly distributed within the range from $1: 20$ to $1: 80$. Moreover, there was no difference in the distribution pattern of the levels between the two groups. On the other hand, although only one patient with pheochromocytoma was available, the chromogranin level in the blood of this patient at a certain stage (26 July, in Table 1) was as high as $1: 640$, which was far higher than the levels in the blood of the normal subjects and the patients with essential hypertension: this finding may be highly evaluated as demonstrating the determination of chromogranin levels in the blood to be useful for the diagnosis of pheochromocytoma. Moreover, the urinary outputs of catecholamines in this patient were never large but small for pheochromocytoma as shown in Table 1 (Yamada, 1969). The fact that the chromogranin level in the blood even of such a patient was high at a certain stage suggests that the determination of chromogranin levels in the blood at given intervals is useful for the diagnosis of pheochromocytoma in the case suspected of this disease, because in the case of small levels with urinary outputs of catecholamines as shown in Table 1, high chromogranin level in blood was observed at a certain stage. But the relation in secretion between catecholamines in $24 \mathrm{hrs}$ urine and chromogranin in blood is yet unclear.

\section{References}

Banks, P (1966). Biochem. J. 101, 536.

Blaschko, H., R. S. Comline, F. Schneider, M. Silver and A. D. Smith (1967). Nature (Lond.) 215, 58.

Douglas, W. W., A. M. Poisner and R. P. Rubin (1965). J. Physiol. 179, 130.

Geffen, L. B., B. G. Livett and R. A. Rush (1969). ibid. 204, 593.

Helle, K. B. New Aspects of Storage and Release Mechanism of Catecholamines (edited by $\mathbf{H}$. J. Schumann and G. Kroneberg) Spring-Verlag, p. 45. (1970).

Helle, K. B. and G. Serck-Hanssen (1975). Mol. Cell Biochem. 6, 127.

Joh, T. H., R. A. Ross and D. J. Reis (1974). Anal. Biochem. 30, 1363.

DeRobertis, E. D. P. and A. VasFerreira (1956). Exp. Cell Res. 12, 568.

Smith, W. J., and N. Kirshner (1967). Mol. Pharmacol. 3, 52.

Yamada, R. (1969). Naika 24, 623. (In Japanese)

Yamada, R. (1975). Rinsyo Kagaku Kensa, Vol. 2. (edited by Y. Ishii) Igaku Syoin p. 534. (1975). (In Japanese)

Yamada, R. and J. Sato (1977). Clinc All Round (Sohgoh Rinsyo) 26, 2414. (In Japanese)

Yamada, R., E. Komiya, J. Sato and T. Nakai (1977). Jap. J. Clin. Pathol. 25, Suppl. 132. (In Japanese)

Yamada, R., J. Sato, E. Komiya and T. Nakai (1978). Endocrinol. Japon. 25, 37. 